\title{
Prensa e historia
}

Por Carlos Ossandón

Se puede sostener que hasta ahora no se ha tematizado suficientemente la interrelación entre comunicación e historia. Esta afirmación no autoriza, sin embargo, a pintar un cuadro desolado, mostrando que nada o muy poco se ha hecho en estos ámbitos. Sería quizá más acertado lamentar el desdén que ha caído sobre unas formas textuales (los periódicos) que no pueden ser ignoradas si se desea destacar perfiles culturales importantes; o subrayar las dificultades de clasificación de un material inmenso, de no siempre fácil consulta, y cuya historicidad está menos a la mano de lo que podría creerse. Tampoco parecería justo defender, y en disputa con determinados celos disciplinarios, la supuesta dignidad de unas formas (y de unos sujetos) subvaloradas, que reclaman ahora su incorporación a la "academia", mereciendo con ello un trato menos correlativo a su propia fugacidad. No parece pertinente buscar afincar aquí la legitimidad de lo que se puede investigar en estos dominios, ni servirse de una estrategia que, advertida según parece por Fredric Jameson, se destruye a sí misma ya que utiliza las armas del adversario; estrategia que consiste en demostrar que los textos o sujetos discriminados son tan "grandes" o importantes como los consagrados por las comunidades hermenéuticas clásicas.

Es cierto, por otra parte, que las perspectivas abiertas por los "Cultural Studies" o, más recientemente, por los "Subaltern Studies", invitan a transitar por escenarios o soportes culturales más amplios que los tradicionales, menos jerarquizados o sujetos a los controles institucionales o epistémicos de los "cánones" (Walter Mignolo), desestabilizando conceptos y prácticas que se creían incuestionadas o sembrando incertezas respecto de lo que parecía importante en el campo cultural (Grinor Rojo). Es igualmente claro que se limpia o queda al menos disculpada la "innobleza" del material que suscita nuestra atención al encajarlo dentro de estas perspectivas problematizadoras o en el marco de unos espacios culturales más híbridos y móviles, cruzados por dimensiones cultas, populares y masivas (Néstor García Canclini).

Entre otros desplazamientos, estas perspectivas han incursionado por tópicos poco trillados, han cuestionado también aquellos relatos anticolonialistas de hace 30 o 40 años atrás, y unas categorías asociadas a unos modos duales u oposicionales de pensar. Gracias a estos y otros impulsos, se han venido sedimentando determinadas ideas-fuerza que de hecho han irradiado más allá de coyunturas específicas o de grupos académicos acotados. Después de todo lo que se ha investigado desde distintas epistemologías en "cultura de masas" ¿quién se atrevería hoy a enarbolar, en los medios académicos especializados, una crítica rigurosamente "culta" o ilustrada a la salsa o al rock urbano? Incluso diagnósticos dualistas que pudieron entusiasmar no hace mucho (como el de fijar una barrera insalvable entre la cultura "letrada" y la de la "imagen") han sido rápidamente disueltos o relativizados por visiones que están más interesadas en describir las nuevas reorganizaciones, modos de leer y descentramientos de los escenarios massmediáticos, que en plantear visiones apocalípticas (Jesús Martín-Barbero). Habiéndose instalado, por otra parte, sospechas importantes sobre las parcelas de saber, las reglas discursivas y los espacios institucionales, concebidos como complejos interpretativos y fábricas de producción de verdades (Michel Foucault) ¿quién arriesgaría un "posicionamiento" de tiempo largo en la defensa de fronteras o de "verdades" definitivas, ahistóricas o fuera de los entramados discursivos? o ¿quien rehusaría sin más la transdisciplinariedad o la necesaria problematización de "la integridad de las disciplinas" (John Beverley), de sus protocolos y clisés?

Como decíamos al comenzar, no es acertado dibujar respecto de los cruces entre comunicación e historia algo parecido a un "campo raso". Y esto ni en el ámbito nacional (Chile) ni tampoco fuera de estas fronteras. Digamos, por de pronto, que lo que se puede llamar genéricamente historia de la comunicación viene experimentando transformaciones importantes que, junto con el desarrollo y la repercusión de las nuevas tecnologías, se expresan en búsquedas que ya no se contentan con narrativas positivistas, en la complejización o ampliación del "objeto", en el establecimiento de vínculos más fluidos entre 
la diversidad de "medios" y el conjunto de la sociedad (ver, por ejemplo, Armand Mattelart: La invención de la comunicación, 1994/1995), en la problematización de las categorías históricas. Aun cuando todavía circulan textos acotados o básicamente descriptivos (como el de Pierre Albert: Historia de la prensa, 1970/1990), o sigue siendo muy útil el voluminoso libro de Georges Weill: El Periódico (1934/1994, 325 pp.), no son éstos los que marcan la agenda.

Por otro lado, parece lógico aceptar que la historiografía comunicacional se ha enriquecido o mezclado con una diversidad de aportes. Las ampliaciones temáticas de la escuela de los "Annales" por ejemplo, su interés por trascender una "histoire événementielle" y exclusivamente política, así como su conocida complejización de los tiempos históricos (Fernand Braudel), son importantes en la dirección que nos interesa (revisar, por ejemplo, las aproximaciones de Jesús Timoteo Alvarez). Cabría mencionar, del mismo modo, las historias de la vida privada (Philippe Ariès y Georges Duby), del libro (Lucien Febvre y Henri-Jean Martin), de las prácticas de lectura (Roger Chartier), de los distintos espacios públicos (Jürgen Habermas, Hannah Arendt, Richard Sennett), etc.; todos desarrollos que le van dando un peso mayor a un campo que ya comienza a exhibir una cierta sensibilidad epistemológica, a transformarse en "problema".

Sin ser todavía probablemente un campo maduro epistemológicamente hablando, la historia de la comunicación puede ya levantar balances o advertir fases o momentos en su desarrollo como saber (ver artículo de Carlos Catalán en Artes y Letras de El Mercurio, 22 marzo 1998). Cuestión que también podemos comenzar a hacer en el ámbito nacional y latinoamericano (revisar los énfasis puestos en esta dirección por Jesús Martín-Barbero).

De hecho, en nuestro país, se cuenta ya con una serie de estudios sobre historia de la comunicación social y más específicamente de la prensa que, aun cuando éstos no se inserten todavía en una "tradición" consciente de sí, capaz ésta de reconocerse en o de demarcarse de su propio "acumulado", permiten emprender la tarea de ordenar y de evaluar dichos estudios.

Se pueden detectar al respecto cuatro enfoques o líneas de investigación. Tenemos, en primer lugar, estudios dedicados a "inventariar" las publicaciones periódicas. Allí están, por ejemplo, los trabajos de Nicolás Anrique Reyes (1904) y de Guillermo Feliú Cruz (1952-1966). Una contribución muy útil en esta línea "inventarial" son las pesquisas de Ramón Briseño, en especial su Cuadro sinóptico periodístico completo de los diarios y periódicos en Chile publicados desde 1812 hasta el año de 1884 (1886), reeditados (1987) en nuevas tablas y con gráficos agregados por Guillermo Martínez y Raymond Colle del Centro de Estudios de la Prensa de la Universidad Católica de Chile. A medio camino entre el inventario y la compilación de artículos se ubican los cuatro tomos sobre los escritos de prensa de Luis Emilio Recabarren, editados por Ximena Cruzat y Eduardo Devés (1985-1987).

Hay, en segundo lugar, un conjunto de trabajos que se pueden agrupar en torno al propósito general de "narrar" las vicisitudes de la prensa nacional, o que usan preferentemente un método descriptivo y empírico a la vez. El libro Prensa y periodismo en Chile (1958) de Raúl Silva Castro lo podemos considerar un "clásico" en esta modalidad. Bueno sería recordar bajo este registro "narrativo" los artículos "pioneros" de Justo Molina (aquel "bibliógrafo ocasional" al decir de Feliú Cruz) aparecidos en La Estrella de Chile en la década de 1870. También los textos de Ricardo Donoso (sobre El Mercurio de Valparaíso y la prensa satírica), Alfonso Valdebenito, Roberto Vilches (sobre las revistas literarias chilenas del XIX), etc.

En tercer lugar, están aquellas publicaciones que tienen una pretensión tan "analítica" como histórica. A diferencia de la anterior orientación, estas publicaciones suelen proponer categorías o sistemas de clasificación del material. En esta línea se pueden mencionar los trabajos de Osvaldo Arias Escobedo (sobre prensa obrera entre 1900 y 1930), Guillermo Sunkel (sobre los diarios populares de masas), Fernando Ossandón (sobre La Epoca y Fortín Mapocho), Patricio Dooner (sobre la prensa durante la Unidad Popular), Santiago Lorenzo (sobre El Mercurio de Valparaíso), Roxana Alvarado (sobre el diario La Cuarta), Carlos Ruiz (sobre El Mercurio y la revista Qué Pasa), Carlos Ossandón (sobre prensa e intelectuales en el siglo XIX), Claudio Durán, Giselle Munizaga, Alfredo Riquelme o las investigaciones auspiciadas por Cerc, 
Céneca e llet. Esta línea de investigación cuenta también con un estudio "clásico": el de Armand Mattelart, Mabel Piccini y Michele Mattelart titulado Los medios de comunicación de masa. La ideología de la prensa liberal en Chile (1970).

Digamos de paso que una serie de trabajos que se han interesado tanto por analizar como por historiar "medios" no escritos, como la radio, el teatro o el cine, no han sido considerados en el orden que exponemos. Tampoco hemos encontrado un lugar adecuado para un trabajo como el de Luis y Fernando Torres (Teoría inconclusa para un continente abierto, 1999), preocupado por historiar y evaluar la vigencia de los distintos modelos comunicacionales en América Latina.

Hay, por último, un cuarto enfoque o línea de investigación que reúne un conjunto dispar de trabajos cuyo punto en común es el de describir o reconstruir tramas comunicacionales globales, desde una perspectiva que combina la mirada histórica con la pregunta por el desarrollo de los procesos de modernización y cambio en Chile. Lo que está en juego en este enfoque es el examen de distintos y espaciados campos de relaciones culturales, así como sus vínculos con la sociedad y la modernización. Autores como Bernardo Subercaseaux, Eduardo Santa Cruz, Gonzalo Catalán o José Joaquín Brunner son reconocibles, desde miradas diversas, en esta línea de investigación.(1)

La clasificación propuesta abre la posibilidad de aventurar al menos dos conclusiones. Digamos, en primer lugar, que los trabajos recientes están claramente más advertidos de las variables conceptuales o metodológicas comprometidas en sus análisis, y no se aprecian recaídas significativas en el positivismo ingenuo. Estructuras o ejes semánticos inspirados en Greimas se constatan en el estudio de A. Mattelart de 1970; matrices y mediaciones culturales en Razón y pasión en la prensa popular de Guillermo Sunkel; bases psicoanalíticas en el examen que hace Claudio Durán del aspecto "ariete" de El Mercurio; "estrategias periodísticas" en los trabajos de Eduardo Santa Cruz; "dispositivos" foucaultianos en los de Carlos Ossandón B. Junto a la construcción de estas y otras "cajas de herramientas", los trabajos más actuales comienzan a pasar menos por alto no sólo las propias "condiciones de existencia" (lenguajes, formatos, etc.) de los periódicos o el carácter fuertemente construido o escénico de éstos (Bernardino M. Hernando), sino también el rol que desempeñan como actores de los procesos modernizadores, así como sus interacciones con otras prácticas sociales y discursivas. La cuestión del espacio público y de la "opinión pública" comienzan a ser igualmente cada vez menos ajenos a unos esfuerzos que ya no conciben a la prensa o a lo comunicacional como meros agregados de una historia reducida a los cauces consabidos.

1 Las demarcaciones establecidas en este afán clasificatorio no son obviamente rígidas, y ciertos autores pueden ser ubicados en más de una línea, o hay fronteras móviles entre, por ejemplo, las dos últimas citadas.

Carlos Ossandón B. es Doctor en Filosofía y Letras. Miembro del Departamento de Investigaciones Mediáticas y de la Comunicación, profesor de la Escuela de Periodismo y del Magister en Comunicación Social de la Universidad de Chile. También investigador y profesor en la U. Arcis. 